\title{
Early Cosmological Period of QCD Confinement
}

\author{
Seyda Ipek and Tim M. P. Tait \\ Department of Physics and Astronomy, University of California, Irvine, California 92697-4575, USA
}

(Received 21 November 2018; published 19 March 2019)

\begin{abstract}
If the strong coupling is promoted to a dynamical field-dependent quantity, it is possible that the strong force looked very different in the early Universe. We consider a scenario in which the dynamics is such that QCD confines at high temperatures with a large dynamical scale, relaxing back to $\sim 1 \mathrm{GeV}$ before big bang nucleosynthesis. We discuss the cosmological implications and explore potential applications, including fleshing out a new mechanism for baryogenesis which opens up if QCD confines before the electroweak phase transition of the standard model.
\end{abstract}

DOI: 10.1103/PhysRevLett.122.112001

Introduction.-The standard model (SM) of particle physics provides a fantastic description of a plethora of low energy observations. That said, it remains incomplete, and experimental probes to date have been largely limited to low energies and temperatures. As a result, it is an intriguing possibility that there could be new physics operating at early cosmological times when the Universe was much hotter than it is today.

The SM predicts that QCD deconfines at temperatures $T \gtrsim \mathrm{GeV}$ and the electroweak symmetry is restored at temperatures $T \gtrsim 100 \mathrm{GeV}$. But our precise understanding of the cosmological history becomes fuzzy for temperatures $T \gtrsim 10 \mathrm{MeV}$ when big bang nucleosynthesis (BBN) begins [1]. It is thus entirely possible that there is physics beyond the SM that produces a radical departure from the standard cosmological picture at earlier times.

Given this blind spot, we ask the question: what if the scale of QCD confinement was itself varying in the early Universe, settling down to the $\sim \mathrm{GeV}$ value we observe today sometime before BBN? We introduce dynamics which allow us to explore this possibility, and map out the consequences for cosmology, the electroweak phase transition, and an opportunity to explain the baryon asymmetry of the Universe.

Dynamical QCD coupling.-In order to promote the QCD coupling to a dynamical quantity, we introduce a scalar field $\phi$, taken to be a SM singlet, coupled to the gluon field strength $G^{\mu \nu}$ via

$$
\mathcal{L} \supset-\frac{1}{4}\left(\frac{1}{g_{s 0}^{2}}+\frac{\phi}{M_{*}}\right) G_{\mu \nu} G^{\mu \nu},
$$

Published by the American Physical Society under the terms of the Creative Commons Attribution 4.0 International license. Further distribution of this work must maintain attribution to the author(s) and the published article's title, journal citation, and DOI. Funded by SCOAP ${ }^{3}$. where $g_{s 0}$ is the QCD coupling for $\langle\phi\rangle=0$, and $M_{*}$ encodes the short distance physics mediating the interaction. $\phi$ could represent fluctuations in a radion or a dilaton, or could represent a generic scalar field which couples to gluons via a triangle diagram containing heavy vectorlike colored particles. In that case $M_{*} \sim 4 \pi M_{Q} / n_{Q} y_{Q} \alpha_{s}$, where $n_{Q}$ is the number of colored fermions with mass $M_{Q}$ and Yukawa coupling $y_{Q}$. It would be interesting to explore the details of the UV dynamics in more detail, but we leave that for future work.

Strong coupling and the QCD scale: When $\phi$ acquires a vacuum expectation value (VEV), it renormalizes the wave function of the gluons, modifying the effective strong coupling. In addition, the coupling $g_{s 0}$ runs with the renormalization scale $\mu$ in the usual way. At one loop,

$$
\frac{1}{\alpha_{s}(\mu,\langle\phi\rangle)}=\frac{33-2 n_{f}}{12 \pi} \ln \left(\frac{\mu^{2}}{\Lambda_{0}^{2}}\right)+4 \pi \frac{\langle\phi\rangle}{M_{*}},
$$

where $n_{f}$ is the number of quark flavors with masses $m_{f} \ll \mu$, and the scale $\Lambda_{0}$ encodes the value of $g_{s 0}$ at some UV reference scale.

Confinement is triggered at the scale $\Lambda$ for which $\alpha_{s}^{-1}(\Lambda,\langle\phi\rangle) \simeq 0$,

$$
\Lambda(\langle\phi\rangle)=\Lambda_{0} \exp \left(\frac{24 \pi^{2}}{2 n_{f}-33} \frac{\langle\phi\rangle}{M_{*}}\right) .
$$

For $n_{f}=6, \Lambda_{0} \sim \mathrm{GeV}$ and $\langle\phi\rangle / M_{*}=-0.62$, QCD confines at $\Lambda \sim 1 \mathrm{TeV}$, well above the temperature of the usual electroweak phase transition.

$\phi$ Potential: We consider a generic potential for $\phi$ at zero temperature,

$$
V(\phi)=\alpha_{1} \phi+\alpha_{2} \phi^{2}+\alpha_{3} \phi^{3}+\alpha_{4} \phi^{4}+\beta_{1} \phi h^{\dagger} h+\beta_{2} \phi^{2} h^{\dagger} h,
$$


where $\alpha_{i}$ and $\beta_{i}$ are couplings with appropriate mass dimension. We have included a trilinear and mixed quartic with the SM Higgs doublet $h$, which would modify the $\phi$ dynamics, and lead to mixing between $\phi$ and the SM Higgs doublet, and are bounded by LHC measurements [2]. Since such interactions do not change the qualitative picture, we simplify the analysis by assuming that they are negligibly small.

When QCD confines, the interaction in Eq. (1) further induces a nonperturbative contribution to $V(\phi)$ via the gluon condensate, $\langle G G\rangle \propto \Lambda^{4}(\langle\phi\rangle)$ [3]. For potentials with a sizable $\alpha_{1}$, this effect is typically unimportant. For example, a benchmark point with $\alpha_{1}=\mathrm{TeV}^{3}, \alpha_{2}=\mathrm{TeV}^{2}$, $\alpha_{3}=\mathrm{TeV}, \alpha_{4}=0.1$, and $M_{*} \sim 13 \mathrm{TeV}$ results in $\langle\phi\rangle \sim-7$ and $m_{\phi} \sim 4 \mathrm{TeV}$, with a negligible contribution from the gluon condensate.

Temperature dependence: In order to restore ordinary QCD with $\Lambda \sim 1 \mathrm{GeV}$ at low temperatures, $\langle\phi\rangle$ must shift by an amount of order $\sim 1 / 2 M_{*}$. The details of how this occurs depend sensitively on both the zero temperature $V(\phi)$ and the finite temperature corrections to it. Whatever the mechanism, it is clear that successfully realizing the $\mathrm{BBN}$ predictions for the primordial abundances of the light elements requires that the temperature at which $\Lambda$ reaches $\Lambda_{\mathrm{QCD}}$ satisfy $T_{\text {res }} \gtrsim 10 \mathrm{MeV}$.

There are a number of constructions which could accomplish this temperature dependence. (i) There may be species (which could be SM singlets) in the plasma with significant coupling to $\phi$, contributing to its effective mass $\propto g^{2} T^{2}$. Such species could play an important role in $\phi$ phenomenology. (ii) If Eq. (1) is generated by vectorlike quarks, there may be additional temperature-dependent contributions to their masses, suppressing the interaction at low temperatures. (iii) There could be a scalar field $\psi$ with its own coupling $\left(\psi / M_{*}\right) G G$, but whose potential induces a positive VEV with parameters tuned to partially cancel the $\phi$ contribution. (iv) Additional scalar fields could couple to $\phi$, and themselves undergo symmetry breaking at low temperatures, triggering a shift in the effective $V(\phi)$ (see, e.g., Ref. [4]). For example, one could introduce two real singlet scalar fields $\phi$ and $\psi$ with the zero-temperature potential

$$
\begin{aligned}
V(\phi, \psi)= & \alpha_{1} \phi+\alpha_{2} \phi^{2}+\alpha_{3} \phi^{3}+\alpha_{4} \phi^{4} \\
& +\beta_{1} \psi^{2}+\beta_{2} \psi^{4}+\gamma_{1} \phi \psi^{2}+\gamma_{2} \phi^{2} \psi^{2},
\end{aligned}
$$

which is invariant under $Z_{2}$ symmetry $\psi \rightarrow-\psi$. For an appropriate choice of parameters, at high temperatures the fields $\phi$ and $\psi$ have zero VEVs. As the universe cools down, first $\phi$ acquires a VEV, and then at a lower temperature, $\psi$ acquires a VEV, which triggers the transition $\langle\phi\rangle \rightarrow 0$.

In the remainder of this work, we remain agnostic concerning the nature of the physics that provides the

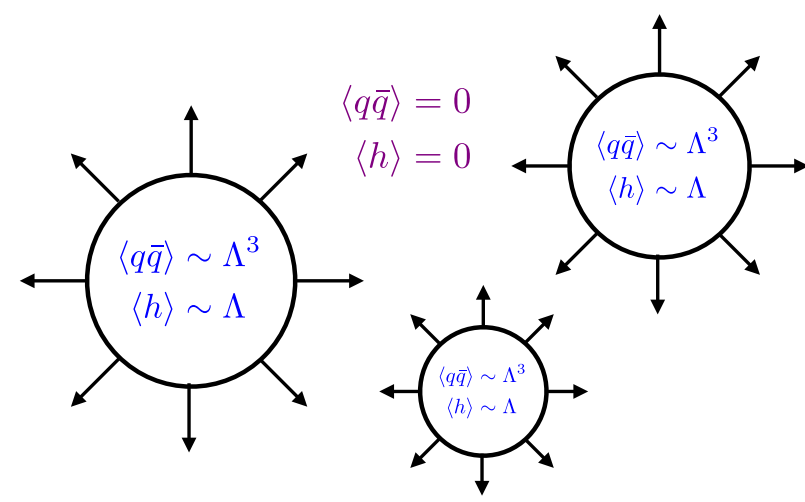

FIG. 1. Bubbles of confined QCD phase are generated and expand. Inside the bubble, the Higgs doublet acquires a VEV due to the tadpole term induced via the quark condensate.

necessary temperature dependence in $V(\phi)$, simply assuming that some mechanism restores standard QCD somewhere in the range $\mathrm{GeV} \lesssim T_{\text {res }} \lesssim 100 \mathrm{GeV}$.

Phase transition and electroweak symmetry breaking.If $S U(3)$ confines before the electroweak phase transition, it triggers electroweak symmetry breaking via chiral symmetry breaking. For $n_{f}=6$ massless quark flavors, the chiral phase transition is expected to be strongly first order $[5,6]$, and proceeds by nucleating bubbles of confined phase with $\langle\bar{q} q\rangle \neq 0$, which expand to fill the Universe. The chiral condensate also couples to the Higgs doublet via the quark Yukawa interactions, appearing as a tadpole which induces a Higgs VEV inside the bubbles. This picture is illustrated in cartoon form in Fig. 1.

The precise details of the QCD phase transition, bubble nucleation, bubble profile, and expansion, are nonperturbative and beyond the scope of this work $[7,8]$. Here, we model the dynamics by a linear sigma model reflecting the approximate $S U(6)_{L} \times S U(6)_{R}$ flavor symmetry of QCD, which is explicitly broken by the SM Yukawa interactions. The field $\Pi(x)$ is a $6 \times 6$ complex scalar containing the pions, scalar mesons, and chiral symmetrybreaking VEVs which is taken to transform under $S U(6)_{L} \times S U(6)_{R}$ as

$$
\Pi(x) \rightarrow L \Pi(x) R^{\dagger},
$$

where $L$ and $R$ are $S U(6)_{L, R}$ transformations, respectively. [Note that the electroweak $S U(2)_{W}$ is a subgroup of $S U(6)_{L}$.] Below the confinement scale, the dynamics of QCD can be described by an effective field theory containing $\Pi(x)$ and the baryons (that are not important for this discussion),

$$
\begin{aligned}
\mathcal{L}_{\Pi}= & \operatorname{Tr}\left[\partial^{\mu} \Pi^{\dagger} \partial_{\mu} \Pi\right]+\mu^{2} \operatorname{Tr}\left[\Pi^{\dagger} \Pi\right]-\lambda_{2} \operatorname{Tr}\left[\Pi^{\dagger} \Pi\right]^{2} \\
& -\lambda_{1} \operatorname{Tr}\left[\Pi^{\dagger} \Pi \Pi^{\dagger} \Pi\right]+\frac{1}{M^{2}} \operatorname{Det} \Pi+\text { H.c. }
\end{aligned}
$$


Naive dimensional analysis (NDA) [9] suggests that up to $\mathcal{O}(1)$ numbers, $\mu^{2} \sim \bar{\Lambda}^{2}, M \sim \bar{\Lambda} / g^{2}$, and $\lambda_{1} \sim \lambda_{2} \sim g^{2}$, where $g \sim 4 \pi$ and $\bar{\Lambda}$ is the cutoff of the chiral effective theory (typically of the order of the $\rho$-meson mass.) Neglecting the SM Yukawa interactions, this would result in a VEV for $\Pi$, breaking $S U(6)_{L} \times S U(6)_{R} \rightarrow S U(6)_{D}$, of the form $\left\langle\Pi_{j}^{i}\right\rangle=f_{\pi} \delta_{j}^{i}$ where $f_{\pi}^{2} \sim \bar{\Lambda}^{2} / g^{2}$.

The explicit $S U(6)_{L} \times S U(6)_{R}$ breaking from the quark Yukawa interactions can be included by treating $Y=y h$ as a spurion, where $h$ is the neutral $C P$ even component of the Higgs doublet, and in the diagonal quark mass basis $y$ is the $6 \times 6$ diagonal matrix whose entries are the quark Yukawa interactions. The corresponding terms containing the spurion $Y$ read,

$$
\begin{aligned}
\mathcal{L}_{Y}= & \left(\tilde{m}^{2} \operatorname{Tr}\left[\Pi^{\dagger} Y\right]+\text { H.c. }\right)-\tilde{\lambda}_{1} \operatorname{Tr}\left[\Pi^{\dagger} Y \Pi^{\dagger} Y\right] \\
& -\tilde{\lambda}_{2} \operatorname{Tr}\left[\Pi^{\dagger} Y Y^{\dagger} \Pi\right]-\tilde{\lambda}_{3} \operatorname{Tr}\left[\Pi Y^{\dagger} Y \Pi^{\dagger}\right],
\end{aligned}
$$

with NDA estimates $\tilde{m}^{2} \sim \bar{\Lambda}^{2} / g, \tilde{\lambda}_{1} \sim \tilde{\lambda}_{2} \sim \tilde{\lambda}_{3} \sim 1$. The first term expands into a tadpole for $h$ induced by the nonzero $\langle\bar{q} q\rangle$ condensate. The remaining terms induce masses (The pion masses in a given epoch scale with both $\Lambda$ and $\langle h\rangle$.) for the 35 pions and induce a backreaction where the Higgs VEV reduces the corresponding chiral condensate by producing a mass for the SM quarks. In principle, this results in a complicated set of coupled equations; however a simple estimate provides a heuristic picture.

The most important entry in $Y$ is the 66 entry corresponding to the top quark with $y_{66}=y_{t} \sim 1$. Including the tadpole generated by $\left\langle\Pi_{66}\right\rangle$ in the finite temperature Higgs potential gives

$$
\begin{aligned}
V_{\mathrm{eff}}(h, T) \simeq & -\frac{\bar{\Lambda}^{2}}{4 \pi} y_{t}\left\langle\Pi_{66}\right\rangle h+\left(\frac{\alpha}{24}\left(T^{2}-T_{c}^{2}\right)\right) h^{2} \\
& -\gamma T h^{3}+\lambda h^{4},
\end{aligned}
$$

where

$$
\begin{aligned}
\alpha & =\sum_{\text {bosons }} g_{i}\left(\frac{m_{i}}{v}\right)^{2}+\frac{1}{2} \sum_{\text {fermions }} g_{i}\left(\frac{m_{i}}{v}\right)^{2}, \\
T_{c} & =2 v \sqrt{\frac{6 \lambda}{\alpha}}, \quad \gamma=\frac{\sqrt{2}}{6 \pi} \sum_{\text {bosons }} g_{i}\left(\frac{m_{i}}{v}\right)^{3},
\end{aligned}
$$

and the sums go through all massive bosons and fermions in the SM. Taking $\left\langle\Pi_{66}\right\rangle \sim \bar{\Lambda} / 4 \pi$, the Higgs VEV can be well approximated by

$$
v \simeq \frac{\bar{\Lambda}}{4}\left(\frac{y_{t}}{\pi^{2} \lambda}\right)^{1 / 3} .
$$

For $\Lambda \sim 1 \mathrm{TeV}$ and taking $\bar{\Lambda}=4 \mathrm{TeV}$, the resulting Higgs VEV is $v \simeq 0.9 \mathrm{TeV}$, as can be seen in Fig. 2 .

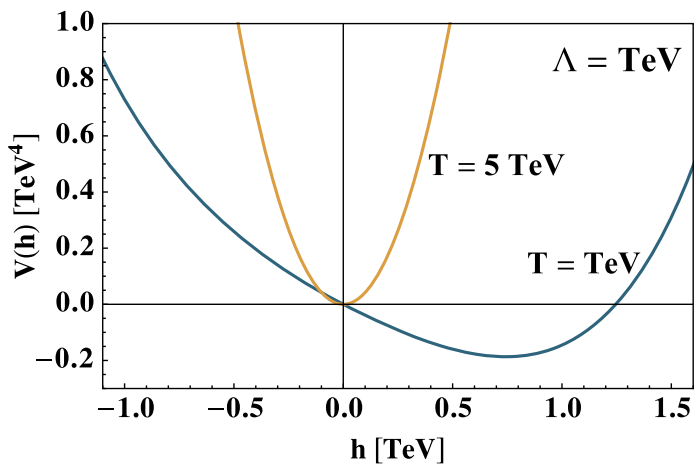

FIG. 2. Finite-temperature Higgs potential at $T=1$ and $5 \mathrm{TeV}$, where the chiral effective theory cutoff is taken to be $\bar{\Lambda}=4 \mathrm{TeV}$.

Applications.—An early period of QCD confinement can have profound implications for the history of the early Universe. We flesh out one particularly exciting possibility to generate the observed baryon asymmetry of the Universe, and sketch several more which would be worth following up in future work below.

Baryogenesis: QCD confining at $\sim \mathrm{TeV}$ temperatures combined with the axion as a solution to the strong $C P$ problem allows for a novel mechanism to explain the baryon asymmetry of the Universe. As mentioned above, since confinement at a $\mathrm{TeV}$ scale takes place when all six of the SM quarks are massless, the phase transition is expected to be first order $[5,6]$ and proceeds through bubble nucleation. Inside the bubble QCD is confined and the EW symmetry is broken (and thus baryon-number violation through the weak interaction is inoperative), whereas outside remains in the unbroken and unconfined phases. Furthermore, if there exists an axion field that addresses the strong $C P$ problem, there can be large $C P$ violation from the uncancelled strong phase during this phase transition $[10,11]$.

The axion couples to the baryon current through the interactions of the pseudoscalar $\eta^{\prime}$ meson, whose mass scales like $m_{\eta^{\prime}} \sim \Lambda$. At energies lower than the $\eta^{\prime}$ mass, its residual effects are described by the effective Lagrangian:

$$
\mathcal{L}_{\mathrm{eff}} \simeq \frac{10}{f_{\pi}^{2} m_{\eta^{\prime}}^{2}} \frac{\alpha_{s}}{8 \pi} G \tilde{G} \frac{\alpha_{w}}{8 \pi} W \tilde{W}
$$

where $W(\tilde{W})$ is the $S U(2)_{W}$ (dual) field strength. As the axion rolls to its minimum, there is an uncanceled $\bar{\theta}$ which induces a $G \tilde{G}$ condensate [10],

$$
\frac{\alpha_{s}}{8 \pi}\langle G \tilde{G}\rangle=m_{a}^{2}(T) f_{a}^{2} \sin \bar{\theta}(T) .
$$

Through the anomaly equation, $\partial_{\mu} j_{B}^{\mu}=\alpha_{W} / 8 \pi W \tilde{W}, W \tilde{W}$ is related to the baryon current density $j_{B}^{\mu}$. Integrating by parts produces an effective chemical potential $\mu$ for baryons: 


$$
\mu=\frac{d}{d t}\left(\frac{10}{f_{\pi}^{2} m_{e \eta^{\prime}}^{2}} m_{a}^{2}(T) f_{a}^{2} \sin \bar{\theta}(T)\right) .
$$

The nonzero chemical potential drives production of a nonzero baryon asymmetry by the electroweak sphalerons,

$$
n_{B}=\int_{T_{i}}^{T_{f}} d t \frac{\Gamma_{\mathrm{sph}}(T)}{T} \mu,
$$

where $\Gamma_{\mathrm{sph}}(T) \sim 25 \alpha_{w}^{5} T^{4}$ is the thermal sphaleron rate outside the bubble where $\langle h\rangle=0$. We assume that inside the bubble the sphalerons are sharply switched off. Making use of the temperature-dependent axion mass [12]

$$
m_{a}^{2}(T) \simeq m_{a}^{2}(T=0) \times\left(\frac{\Lambda}{T}\right)^{7},
$$

where

$$
m_{a}^{2}(T=0) \sim m_{\pi}^{2} \frac{f_{\pi}^{2}}{f_{a}^{2}}
$$

assuming $\sin \bar{\theta}$ is varying slowly, and $\Delta m_{a}^{2}\left(T_{\mathrm{PT}}\right) \simeq$ $m_{a}^{2}\left(T_{\mathrm{PT}}\right)$ around $T_{\mathrm{PT}}$, the temperature at which the EW transition happens, the resulting baryon-to-entropy ratio is

$\eta=\frac{n_{B}}{s} \simeq \frac{45 \times 125}{2 \pi^{2} g_{*}\left(T_{\text {reh }}\right)} \frac{m_{\pi}^{2}}{m_{\eta^{\prime}}^{2}} \alpha_{w}^{5} \sin \bar{\theta}\left(\frac{T_{\mathrm{PT}}}{T_{\text {reh }}}\right)^{3}\left(\frac{\Lambda}{T_{\mathrm{PT}}}\right)^{7}$,

where $T_{\text {reh }}$ is the reheat temperature at the end of the EW phase transition and $g_{*}$ counts the relativistic degrees of freedom at that time. This picture has all of the dynamics naturally occurring concurrently, resulting in $T_{\text {reh }} \simeq T_{\mathrm{PT}} \simeq$ $\Lambda$ and in the baryon asymmetry being roughly independent of the temperature at which QCD confines provided this happens above the electroweak scale. The baryon-toentropy ratio is

$$
\eta \simeq 1.8 \times 10^{-9} \times \sin \bar{\theta},
$$

to be compared with the Planck measurement [13],

$$
\eta_{\text {exp }}=(8.59 \pm 0.11) \times 10^{-11} .
$$

Achieving the observed baryon asymmetry requires a very modest tuning of $\sin \bar{\theta} \sim 1 / 10$ or a small amount of dilution after the baryon asymmetry is generated. It is remarkable that the baryon asymmetry is naturally close to the observed value for $\bar{\theta}$ of order 1 , despite the relative dearth of adjustable parameters.

Other applications: The baryon asymmetry is just one application of an early period of QCD confinement out of many that could be imagined. We leave detailed follow up for future work, but a few others would be the following:
$B B N$ and early Universe.-Early QCD confinement could leave an imprint on BBN if the transition to $\Lambda \sim$ $1 \mathrm{GeV}$ occurs late enough, implying bounds on the dynamics of $\phi$. Furthermore, while confined the SM plasma degrees of freedom are different, influencing the evolution of the Universe.

Dark matter freeze-out.-If dark matter freezes-out during a period in which QCD is confined, the relevant degrees of freedom both for annihilation and in the plasma correspond to the confined phase, in contrast with usual WIMP scenarios.

Axion dark matter.-For theories invoking a QCD axion, the early period of confinement switches on the axion potential earlier and generates a larger axion mass (of order $10^{4}$ the usual mass for $\Lambda \sim \mathrm{TeV}$ ). For very large $\Lambda$, the axion could decay on cosmological timescales, erasing its density. Even for more modest $\Lambda$, the transition to $\Lambda \sim 1 \mathrm{GeV}$ would induce a novel temperature dependence on the axion mass, and could, e.g., result in an early period of matter domination.

Gravitational waves. - As with other first order cosmological phase transitions, the early period of QCD confinement is expected to generate gravitational waves $[14,15]$. The detailed predictions will depend sensitively on the dynamics of the bubble nucleation, expansion, and collision, which themselves take place in a background of the strongly interacting plasma (which could, for example, provide friction slowing down the bubble expansion rate). A careful investigation of the properties of the phase transition and its impact on gravitational wave production is currently under investigation [16].

Collider searches. - The most model-independent prediction is the existence of the neutral scalar field $\phi$ which couples to gluons, and could contribute to dijet signatures at high energy colliders. If it mixes with the Higgs doublet, it will pick up other couplings to SM fields, and induce deviations in the Higgs couplings. There may be additional neutral or colored particles coupled to $\phi$ as well.

Heavy ion collisions. - If the dynamics restoring $\Lambda \sim$ $1 \mathrm{GeV}$ occur at low energies, there are likely to be indications visible in high temperature environments such as heavy ion collisions.

$S U(2)_{W}$ confinement.-A similar mechanism could be employed, e.g., to trigger $S U(2)_{W}$ confinement in the early Universe, with interesting consequences for electroweak symmetry breaking and potentially opening more new avenues for baryogenesis.

Conclusions and outlook.-Given our lack of knowledge about QCD at high temperatures, it is natural to ask whether there may be surprising dynamics which were important in the early Universe, but remain hidden at low temperatures. If QCD confines at a high scale, returning to $\Lambda \sim 1 \mathrm{GeV}$ at later times, it may shed light on some of the mysteries of our Universe, including the fact that it is made out of matter and not antimatter. We have sketched the basic properties of 
such a scenario, and demonstrated that baryogenesis can work if there is an axion that solves the strong $C P$ problem. Many open questions remain open, and many avenues remain to be explored in this framework.

We are grateful for discussions with Djuna Croon, Anna Hasenfratz, Christopher T. Hill, Ciaran Hughes, David B. Kaplan, David McKeen, Michael Ramsey-Musolf, and Geraldine Servant. This work is supported in part by NSF Grant No. PHY-1620638. S. I. acknowledges support from the UC Office of the President via a UC Presidential Postdoctoral fellowship. This work was performed in part at Aspen Center for Physics, which is supported by National Science Foundation Grant No. PHY-1607611.

[1] M. Tanabashi et al. (Particle Data Group), Phys. Rev. D 98, 030001 (2018).

[2] G. Aad et al. (ATLAS and CMS Collaborations), J. High Energy Phys. 08 (2016) 045.
[3] B. von Harling and G. Servant, J. High Energy Phys. 01 (2018) 159.

[4] M. J. Baker and J. Kopp, Phys. Rev. Lett. 119, 061801 (2017).

[5] R. D. Pisarski and F. Wilczek, Phys. Rev. D 29, 338 (1984).

[6] H.-T. Ding, J. Phys. Conf. Ser. 432, 012027 (2013).

[7] K. Kajantie, Phys. Lett. B 285, 331 (1992).

[8] Y. Bai, A. J. Long, and S. Lu, arXiv:1810.04360.

[9] A. G. Cohen, D. B. Kaplan, and A. E. Nelson, Phys. Lett. B 412, 301 (1997).

[10] V. A. Kuzmin, M. E. Shaposhnikov, and I. I. Tkachev, Phys. Rev. D 45, 466 (1992).

[11] G. Servant, Phys. Rev. Lett. 113, 171803 (2014).

[12] M. S. Turner, Phys. Rev. D 33, 889 (1986).

[13] P. A. R. Ade et al. (Planck Collaboration), Astron. Astrophys. 594, A13 (2016).

[14] C. Caprini et al., J. Cosmol. Astropart. Phys. 04 (2016) 001.

[15] D. Cutting, M. Hindmarsh, and D. J. Weir, Phys. Rev. D 97, 123513 (2018).

[16] D. Croon, S. Ipek, and T. M. Tait (to be published). 